\title{
Vitamin B12 deficiency in patients with type 2 diabetes mellitus using metformin and the associated factors in Saudi Arabia
}

Roqaih R. Al Saeed, MBBS, SBFM, Muneera A. Baraja, SBFM, ABFM.

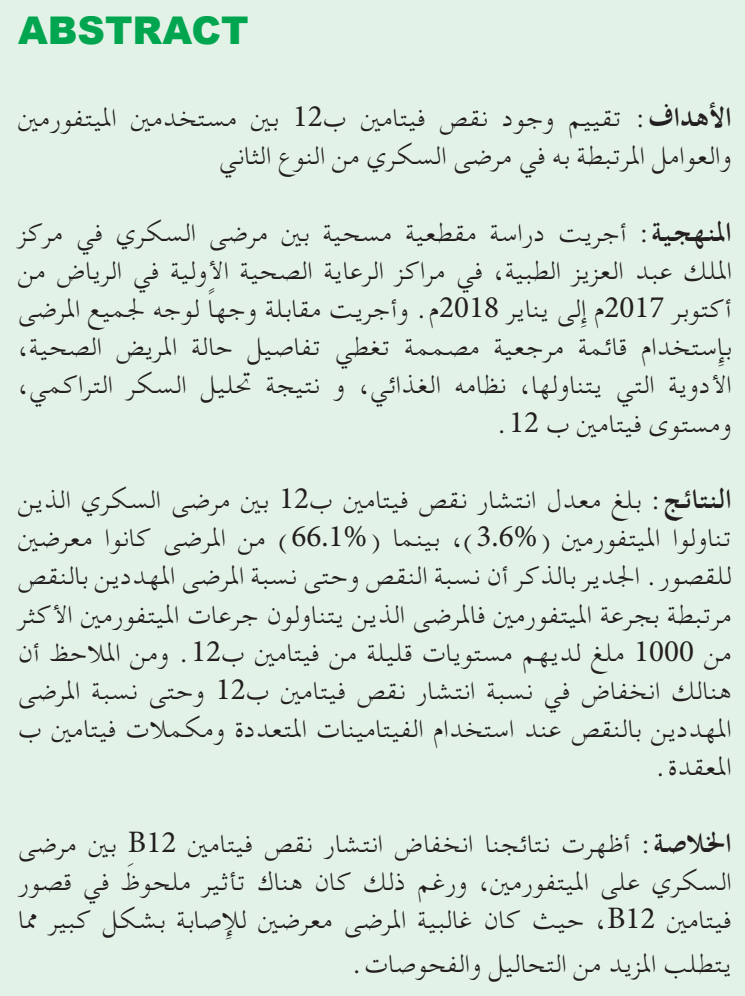

Objectives: To assess the presence of vitamin B12 deficiency among metformin users and associated factors in patients with type 2 diabetes mellitus.

Methods: An observational cross-sectional study was conducted with 307 diabetic patients in the primary health care centers at King Abdulaziz Medical City (KAMC), Riyadh, Saudi Arabia between October 2017 and January 2018. Face-to-face interviews were conducted with the participants, using a designed checklist.

Results: The prevalence of vitamin B12 deficiency was (3.6\%) and the majority of the sample had borderline B12 levels (66.1\%). The vitamin B12 deficiency and borderline levels were strongly associated with the dose of metformin. Patients taking doses of metformin more than $1000 \mathrm{mg}$ had lower levels of vitamin B12. The use of multivitamin and vitamin B complex was assessed, and it was found that there was a marked decrease in the prevalence of vitamin B12 deficiency in patients using vitamin $\mathrm{B}$ complex containing more than $200 \mathrm{mcg}$ vitamin B12.

Conclusion: Our findings show a low prevalence of vitamin B12 deficiency in type 2 diabetic patients taking metformin. The majority of the sample had borderline deficiency; therefore, additional investigation and management are required.

Keywords: type 2 diabetes mellitus, metformin, vitamin B12 deficiency

Saudi Med J 2021; Vol. 42 (2): 161-165

doi: 10.15537/smj.2021.2.25693

From the Department of Family Medicine (Al Saeed, Baraja), King Abdullah International Medical Research Center/King Saud bin Abdulaziz University for Health Sciences, King Abdulaziz Medical City, Ministry of National Guard Health Affairs, Riyadh, Kingdom of Saudi Arabia.

Received 11th October 2020. Accepted 30th December 2020.

Address correspondence and reprint request to: Dr. Roqaih R. Al Saeed, Department of Family Medicine, King Abdullah International Medical Research Center/King Saud bin Abdulaziz University for Health Sciences, King Abdulaziz Medical City, Ministry of National Guard Health Affairs, Riyadh, Kingdom of Saudi Arabia. E-mail: roqaih.alsaad@gmail.com ORCID ID: http://orcid.org/0000-0001-9865-5539

Disclosure. Authors have no conflict of interests, and the work was not supported or funded by any drug company. 
$\mathrm{D}$ iabetes mellitus (DM) is a widely prevalent disease managed in primary health care, and its prevalence has increased significantly in the past decade. ${ }^{1}$ It is considered a serious public health concern due to the multiple comorbidities and complications, including retinopathy, nephropathy, peripheral neuropathy, cardiovascular diseases, and the necessity for lifelong management. ${ }^{2}$ According to the American Diabetes Association (ADA), metformin is the initial drug of choice and the cornerstone for the control of type 2 diabetes due to its high efficacy in controlling macro-vascular complications. ${ }^{3}$ However, when used for prolonged periods, it may cause biochemical side effects such as vitamin B12 deficiency. ${ }^{4}$

Several international studies have assessed vitamin B12 deficiency among type 2 diabetic patients using metformin. The prevalence of metabolically confirmed B12 deficiency in type 2 diabetic population on metformin was between $4 \%$ and $41 \% .^{5,6}$ However, there is considerable variation in the studies regarding the factors influencing this association. In a 2017 study conducted in Peshawar, Pakistan, it was found that the use of multivitamins and vitamin B12 supplement could be protective against developing vitamin B12 deficiency. ${ }^{7}$ Another study from Lahore, Pakistan, conducted in 2019, reported that longer duration (more than 2 years) of metformin use was strongly associated with vitamin B12 deficiency. ${ }^{8}$

In Saudi Arabia and the Middle East, there are large number of cases of diabetes and its associated complications, but there is limited research regarding vitamin B12 deficiency related to the use of metformin. The aim of this study is to assess the presence of vitamin B12 deficiency among metformin users in patients with type 2 diabetes mellitus. The sample of patients were drawn from the primary health care centers at King Abdulaziz Medical City (KAMC), Riyadh, Saudi Arabia. The study also attempts to analyze the effect of supplementation on B12 levels.

Methods. From October 2017 to January 2018, a cross-sectional study was undertaken with type 2 diabetic patients at KAMC primary health care centers in Riyadh. Face-to-face interviews were conducted using a designed checklist to collect demographic data, patient condition, medication, diet, glycated hemoglobin, and vitamin B12 level. The inclusion criteria for this study were: participant had to be a type 2 diabetic patient between the ages of 18 and 80 years; taking an oral antidiabetic medication, including metformin, for more than 3 months; and attending a KAMC primary health care center in Riyadh. The exclusion criteria included type 1 diabetic patients; patients on an insulin-only regimen; those having a chronic kidney disease defined by a creatinine level $>3.0 \mathrm{mg} / \mathrm{dl}$; patients suffering from chronic liver disease, Crohn's disease, and thyroid disease; patients who had undergone gastric or bariatric surgery or ileum resection; pregnant women, severely ill patients who were unable to give consent, and mentally ill patients.

A convenience sampling method was used in this study. Patients with type 2 diabetes were notified about the study, and an informed consent was obtained from them. The sampling was carried out 3 days per week, and 5 to 7 patients were interviewed during a regular scheduled visit to the outpatient clinic. A blood sample was taken from each patient to measure the level of vitamin $\mathrm{B} 12$ and $\mathrm{HbA} 1 \mathrm{c}$ within a month of the interview. There is multiple blood test usually used to aid the diagnosis of vitamin B12 deficiency are homocysteine, serum vitamin B12, and plasma methylmalonic acid. ${ }^{9}$ As these tests are not gold standard tests, it is difficult to establish a clear cutoff point as a diagnostic value. Serum cobalamin is the most frequently used test and considered the initial investigation for vitamin B12 deficiency. The serum vitamin B12 was measured using the ARCHITECT B12 assay. The reference value indicating vitamin B12 deficiency was $<150 \mathrm{pg} / \mathrm{mL}$, a value between 150 and $399 \mathrm{pg} / \mathrm{mL}$ was considered for borderline deficiency, and a value above $400 \mathrm{pg} / \mathrm{mL}$ was considered normal.? The checklist for the interviews was designed and developed by the researcher after an extensive literature review. The checklist was validated by 2 family medicine consultants and translation validity was ensured using the forward and backward translation from English to Arabic.

Participants were included after written informed consent. The study and the informed consent form were approved by the Institutional Review Board (IRB) of King Abdullah International Medical Research Center (KAIMRC) according to the principles of Helsinki Declaration.

Data management and analysis plan. The data were analyzed using the Statistical Package for Social Sciences program, version 23 (IBM Corp., Armonk, N.Y., USA). The categorical variables are expressed in frequency and percentage. A Chi-square was used to assess differences between the categorical variables. Continuous variables were expressed with a mean and standard division. A $p<0.05$ was considered statistically significant.

Results. Three hundred and seven patients were enrolled in the study. The age of the patients ranged from 20 to 79 years, with a mean of $58.69 \pm 11.1$ years. 
The majority $(\mathrm{n}=191 ; 62.2 \%)$ were females and 169 (55\%) had been diagnosed with type 2 diabetes for less than 10 years while 138 (45\%) had been diabetic for more than 10 years. In the majority $(60.9 \%)$ of cases, the diabetes was uncontrolled with a mean of $8.5 \pm 1.68$. Approximately 187 (60.9\%) were using $\geq 1000 \mathrm{mg}$ of metformin and $120(39 \%)<1000 \mathrm{mg}$ of metformin.

Table 1 - Characteristics and demographic data of diabetic patients on metformin $(\mathrm{N}=307)$.

\begin{tabular}{lc}
\hline Characteristics & $\mathbf{n}(\%)$ \\
\hline Gender & $116(37.8)$ \\
$\quad$ Male & $191(62.2)$ \\
$\quad$ Female & \\
Duration of DM (years) & $169(55.0)$ \\
$\quad<10$ years & $138(45.0)$ \\
$\quad \geq 10$ years & \\
Metformin dose & $120(39.1)$ \\
$\quad<1000$ mg & $187(60.9)$ \\
$\quad \geq 1000$ mg & \\
Smoking & $21(6.8)$ \\
$\quad$ Yes & $286(93.2)$ \\
$\quad$ No & \\
Alcohol use & $1(0.3)$ \\
$\quad$ Yes & $306(99.7)$ \\
$\quad$ No & \\
Vegetarian & $0(0)$ \\
Yes & $307(100)$ \\
No & $58.6(11.1)$ \\
Age (years) (mean $\pm S D)$ & $8.5(1.68)$ \\
HbAlc level (mean $\pm S D)$ & $9.53(5.853)$ \\
Length of metformin use (years) (mean $\pm S D)$ & \\
\hline$\quad$ Values are expressed as numbers and percentages $(\%)$. \\
$\quad$ DM: diabetes mellitus. A1C: glycated hemoglobin.
\end{tabular}

The mean duration of metformin use was $9.53 \pm 5.853$ years. The vast majority were nonsmokers (93.2\%) and nonalcoholics $(99.7 \%)$ and none were vegetarians (Table 1).

Eleven (3.6\%) was vitamin B12 deficient $(<150 \mathrm{pg} / \mathrm{mL})$, and 203 (66.1\%) had borderline deficiency $(150-399 \mathrm{pg} / \mathrm{mL})$. Approximately 93 of the samples $(30.3 \%)$ had normal vitamin B12 levels $(\geq 400 \mathrm{pg} / \mathrm{mL})$. Only $11.4 \%$ of the patients were taking multivitamins, and many of these patients $(41.2 \%)$ had been taking it for more than 2 years. More than half $(62.2 \%)$ were taking a vitamin B supplement, and $46.4 \%$ were taking it for more than 2 years.

As shown in Figure 1, the group that was taking $>1000 \mathrm{mg}$ metformin had higher proportions of vitamin B12 borderline (71\%) and deficiency levels (4.3\%), compared to $58.3 \%$ and $2.5 \%$ in the group taking $<1000 \mathrm{mg}$ metformin, which was statistically significant $(p=0.023)$. Higher levels of vitamin B12 deficiency occurred in the group $<60$ years $(n=6 ; 3.9 \%)$ compared to $\geq 60$ years and older $(n=5 ; 3.3 \%)$. However, the borderline deficiency was higher in the group 60 years and older $(\mathrm{n}=111 ; 73 \%)$, which was statistically significant $(p=0.011)$.

The reference value indicating vitamin B12 deficiency was $<150 \mathrm{pg} / \mathrm{mL}$, a value between 150 and $399 \mathrm{pg} / \mathrm{mL}$ was considered for borderline deficiency, and a value of above $400 \mathrm{pg} / \mathrm{mL}$ was considered normal.

For the group that was taking both metformin and vitamin B complex, almost half (45\%) had normal vitamin B12 levels, 45.1\% borderline levels, and $0.9 \%$ were deficient. The association was statistically significant $(p=0.0)$.

Table 2 - Association between vitamin B12 levels and use of a supplement $(\mathrm{N}=307)$.

\begin{tabular}{|c|c|c|c|c|c|}
\hline Supplement use & Deficiency & Borderline & Normal & Chi-square & $P$-value \\
\hline B-complex & & & & 49.22 & $0.00^{*}$ \\
\hline Yes & $1(0.9)$ & $51(45.1)$ & $61(45.0)$ & & \\
\hline No & $10(5.2)$ & $150(78.5)$ & $31(16.2)$ & & \\
\hline Multivitamins & & & & 1.657 & 0.437 \\
\hline Yes & $0(0.0)$ & $23(65.7)$ & $12(34.3)$ & & \\
\hline No & $11(4.1)$ & $180(66.4)$ & $80(29.5)$ & & \\
\hline Duration of B-complex (years) & & & & 2.283 & 0.319 \\
\hline$<2$ & $1(107)$ & $24(40.0)$ & $35(58.3)$ & & \\
\hline$\geq 2$ & $0(0.0)$ & $27(51.9)$ & $25(48.1)$ & & \\
\hline Duration of multivitamins use (years) & & & & 0.273 & 0.458 \\
\hline$<2$ & $0(0.0)$ & $15(75.0)$ & $5(25.0)$ & & \\
\hline$\geq 2$ & $0(0.0)$ & $8(57.6)$ & $6(42.9)$ & & \\
\hline
\end{tabular}




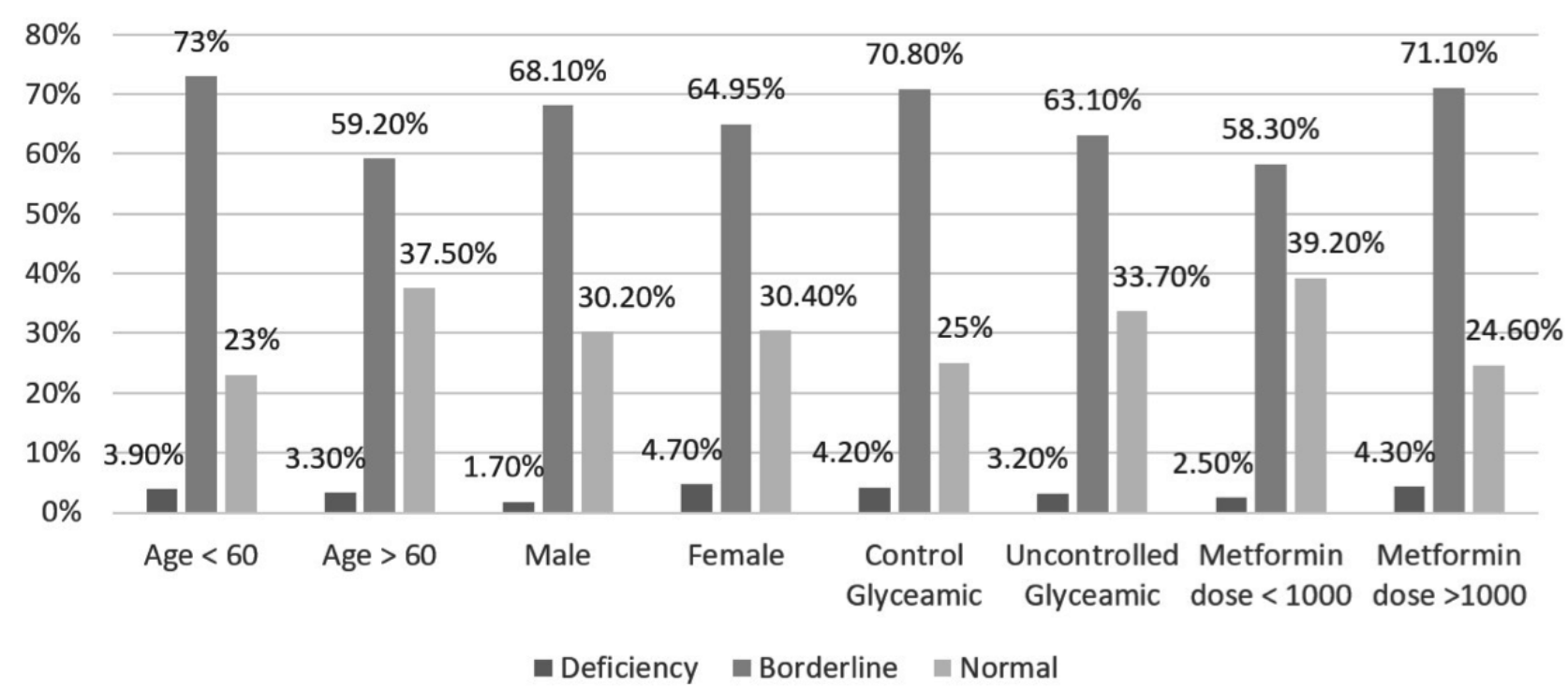

Figure 1 - Relation between patient's characteristics and vitamin B12 levels.

Discussion. In the current study, the prevalence of vitamin B12 deficiency in diabetic patients taking metformin was $3.6 \%$. This finding is similar to two studies carried out in Pakistan and Saudi Arabia, at $4 \%$ and $9.4 \%$, respectively. ${ }^{5,10}$ However, this study is in contrast to a study conducted in South South Nigeria, where the prevalence was $40 \%{ }^{6}$ Possible reasons for the variations in vitamin B12 levels globally are the cutoff levels used in the studies, diverse cultural and religious beliefs, as well as dietary habits, which play a significant role in vitamin B12 levels. The majority (66.1\%) of the sample in this study had borderline B12 levels. This is higher than what was reported (59\%) by the study in South South Nigeria. ${ }^{6}$ Patients with borderline deficiency need additional evaluation to identify the group who are vitamin B12 deficient. The use of homocysteine and methylmalonic acid tests can increase the sensitivity of vitamin B12 deficiency detection; ${ }^{11}$ however, the tests were not available at our facility.

The vitamin B 12 deficiency and borderline levels were not affected by the duration of metformin use but were strongly associated with the dose of metformin. Patients taking higher doses of metformin, >1000 mg, had lower levels of vitamin B12. These results are consistent with several studies where the metformin dose was the strongest predicator of vitamin B12 deficiency, as each increase in metformin dose increases the risk of vitamin B12 deficiency. ${ }^{10,12}$

The use of any supplement containing vitamin B12 was also assessed in this study, and a marked decrease was noted in the prevalence of vitamin B12 deficiency in patients using vitamin B complex with $>200 \mathrm{mcg}$ vitamin B12. However, a multivitamin with $6 \mathrm{mcg}$ vitamin B12 did not change the prevalence of borderline or full-fledged vitamin B12 deficiencies. Moreover, some studies reported that using any dose of vitamin B12 supplement will improve the vitamin B12 levels. ${ }^{7,13,14}$

In addition, the current study did not find a correlation between diabetes control and vitamin B12 deficiency, using the HbA1c levels as the predictor of diabetes control. The same result has been reported in existing literature, and this could be because the effect on the vitamin B12 levels is due to the metformin and not the diabetes itself. 5,10

An assessment of the risk factors that may affect vitamin B12 levels showed that the deficiency occurred less in older patients. The reason could be the use of supplements by older patients, compared to younger diabetic patients. In addition, no gender difference was noticed in the study, which is similar to study from Bangladesh. ${ }^{15}$

Study limitations. First, our study was confined to patients from a single hospital at KAMC in Riyadh, and specifically to the primary health care centers, which make it difficult to generalize the results on Saudi Arabia as a whole. Another limitation of our study is that we used cross-sectional study, and it may not be the best way to establish a causal relationship between vitamin B12 levels and patient factors. Third, homocysteine and methylmalonic acid tests, which play an important role 
in proper identification of patients with true vitamin B12 deficiency, were not performed in this study for patients who exhibited borderline deficiency.

In conclusion, the prevalence of vitamin B12 deficiency in diabetic patients on metformin found to be low in this study. The majority of patients had borderline B12 deficiency, necessitating further investigation and management. The metformin dose was the strongest factor contributing to this deficiency, and it was seen that taking a vitamin B12 supplements prevents the development of vitamin B12 deficiency. Further studies are required to assess vitamin B12 levels in relation to metformin to represent the entire Saudi Arabia population and measure serum homocysteine and methylmalonic acid levels along with vitamin B12 level for better diagnosis and management.

Acknowledgment. We would to like to thank (Scribendi) for the English language editing.

\section{References}

1. World Health Organization. Global report on diabetes. [Update 2018. Accessed 2018 June 7]. Available from: https:// apps.who.int/iris/bitstream/handle/10665/204871/978924156 5257_eng.pdf;jsessionid=CE740C6AF72BCFC9BD19F9C22 4A028D8? sequence $=1$

2. Kakkar R. Rising burden of diabetes-public health challenges $\&$ way out. Nepal J Epidemiol 2016; 6: 557-559.

3. American Diabetes Association. Standards of medical care in diabetes - 2020. Diabetes Care 2020; 43 (Suppl 1): S98-S110.

4. Out M, Kooy A, Lehert P, Schalkwijk CA, Stehouwer CD. Long-term treatment with metformin in type 2 diabetes and methylmalonic acid: post hoc analysis of a randomized controlled 4.3 year trial. J Diabetes Complications 2018; 32: 171-178.

5. Miyan Z, Waris N. Association of vitamin B12 deficiency in people with type 2 diabetes on metformin and without metformin: a multicenter study, Karachi, Pakistan. BMJ Open Diabetes Res Care 2020; 8: e001151.
6. Owhin SO, Adaja TM, Fasipe OJ, Akhideno PE, Kalejaiye OO, Kehinde MO. Prevalence of vitamin B12 deficiency among metformin-treated type 2 diabetic patients in a tertiary institution, South-South Nigeria. SAGE Open Med 2019; 7: 2050312119853433.

7. Khan A, Shafiq I, Hassan Shah M. Prevalence of vitamin B12 deficiency in patients with type II diabetes mellitus on metformin: a study from Khyber Pakhtunkhwa. Cureus 2017; 9: e1577.

8. Hasan NU, Makki MU, Abid I, Abid Butt MU. Association of vitamin B12 deficiency with intake of oral metformin in diabetic patients. J Ayub Med Coll Abbottabad 2019; 31: 72-75.

9. Langan RC, Goodbred AJ. Vitamin B12 deficiency: recognition and management. Am Fam Physician 2017; 96: 384-389.

10. Alharbi TJ, Tourkmani AM, Abdelhay O, Alkhashan HI, Al-Asmari AK, Bin Rsheed AM, et al. The association of metformin use with vitamin B12 deficiency and peripheral neuropathy in Saudi individuals with type 2 diabetes mellitus. PLoS One 2018; 13: e0204420.

11. Oberley MJ, Yang DT. Laboratory testing for cobalamin deficiency in megaloblastic anemia. Am J Hematol 2013; 88: 522-526.

12. Aroda VR, Edelstein SL, Goldberg RB, Knowler WC, Marcovina SM, Orchard TJ, et al. Long-term metformin use and vitamin B12 deficiency in the diabetes prevention program outcomes study. J Clin Endocrinol Metab 2016; 101: 1754-1761.

13. Elsaier A, Elamami AH, Zaid I. Vitamin B12 deficiency in patients with type 2 diabetes on metformin therapy. Libyan J Med 2017; 9: 72-75.

14. Bherwani S, Ahirwar AK, Saumya AS, Sandhya AS, Prajapat B, Patel S, et al. The study of association of vitamin B12 deficiency in type 2 diabetes mellitus with and without diabetic nephropathy in North Indian population. Diabetes Metab Syndr 2017; 11 (Suppl 1): S365-S368.

15. Kamrul-Hasan AB, Fariduddin M, Ghosh DK, Islam M, Atikur-Rahman M, Sultana N, et al. Vitamin B12 is found sufficient in newly diagnosed type 2 diabetes in a hospital based study. Int J Diabetes Metab Disord 2016; 1: 1-7. 